\title{
Linear Polyubiquitination: A Crucial Regulator of NF-кB Activation
}

\author{
Kazuhiro Iwai
}

\begin{abstract}
NF- $\kappa \mathrm{B}$ is a transcription factor known to be involved in pleomorphic biological phenomena such as inflammation and immune responses. Abnormal activation of NF- $\mathrm{KB}$ has been reported in many pathological conditions, including malignant tumors. Therefore, the NF- $\mathrm{KB}$ activation pathway has been extensively studied and involvement of the ubiquitin conjugation system in the NF- $\kappa \mathrm{B}$ activation pathways has been revealed. Although the ubiquitin conjugation system was discovered as a part of a protein degradation pathway, non-degradable roles of the ubiquitin system have been revealed recently. Several types of polyubiquitin chains exist in cells and the type of chain seems to determine how ubiquitinated proteins are regulated. We have identified that a new type of polyubiquitin chain, the linear polyubiquitin chain, plays a crucial role in regulating the $\mathrm{NF}-\kappa \mathrm{B}$ activation pathway in non-degradable manner. In this chapter, the discovery, roles in NF- $\kappa \mathrm{B}$ activation, and involvement in the pathogenesis of cancers of linear ubiquitination will be discussed.
\end{abstract}

Keywords NF- $\kappa$ B $\bullet$ Ubiquitin $\bullet$ LUBAC $\bullet$ Linear ubiquitin chain $\bullet$ cpdm $\bullet$ B cell lymphoma

\section{The Ubiquitin Conjugation System}

The ubiquitin system, which is one of the most extensively studied post-translational protein modification systems, has been identified as part of an energy-dependent degradation system [1]. Ubiquitin is a protein-based modifier composed of 76 amino acids. Through the function of three enzymes called the ubiquitin-activating enzyme (E1), the ubiquitin conjugating enzyme (E2) and the ubiquitin ligase (E3), ubiquitin is conjugated to the substrate proteins that are recognized by E3s. Ubiquitin is added onto ubiquitin pre-conjugated to the substrates to generate polyubiquitin chains - polymer of ubiquitin. The proteasome recognizes the polyubiquitin chains

K. Iwai $(\bowtie)$

Department of Molecular and Cellular Physiology, Graduate School of Medicine,

Kyoto University, Yoshida-konoe-cho, Sakyo-ku, Kyoto 606-8501, Japan

e-mail: kiwai@mcp.med.kyoto-u.ac.jp

K. Nakao et al. (eds.), Innovative Medicine, DOI 10.1007/978-4-431-55651-0_4 
and destines ubiquitinated proteins for degradation (Fig. 1) [2]. The physiological significance of the ubiquitin proteolytic system mainly arises from its timely and selective recognition of the substrate proteins [2]. However, timely and selective protein modification is a desirable feature not only for degradation but also for other modes of protein regulation. As expected, these non-degradation functions of the ubiquitin system are now widely recognized [3]. Among the post-translational modification systems, the ubiquitin system has the unique feature that the system regulates the function of proteins by conjugating polyubiquitin chains in most cases. There are several types of polyubiquitin chains in cells, and the type of polyubiquitin chain is thought to determine the mode of regulation of the conjugated proteins [3]. Polyubiquitin chains have been believed to be exclusively generated via linkage between the carboxyl group of a ubiquitin monomer and $\varepsilon$-amino group of one of seven Lys residues within ubiquitin. For example, ubiquitin chains generated via Lys(K)48 of ubiquitin (K48 chains) function as a degradation signal and chains generated via K63 (K63 chains) are involved in DNA repair or signal transduction without a functioning degradation signal (Fig. 1) [3]. We have identified a new type of polyubiquitin chain, the linear polyubiquitin chain, in which the carboxyl group of a ubiquitin monomer forms a peptide bond with an N-terminal Met residue of another ubiquitin molecule (Fig. 1) [4]. We have also identified the ubiquitin ligase
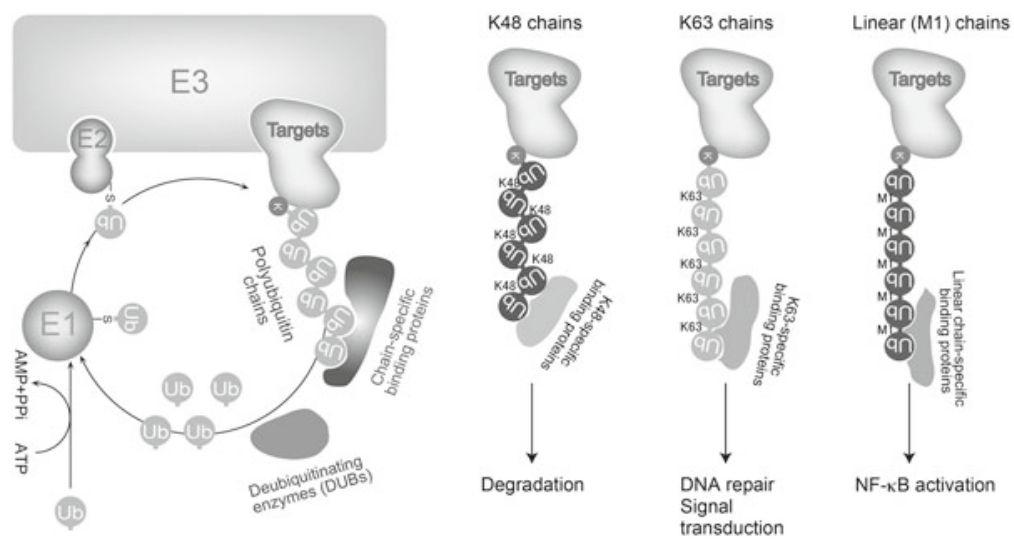

Fig. 1 The ubiquitin conjugation system. Through the function of three enzymes, called ubiquitin activating enzymes (E1), ubiquitin conjugating enzymes (E2), and ubiquitin ligases (E3), ubiquitin is conjugated to target proteins that are specifically recognized by E3s. Once an ubiquitin is conjugated to the target protein, additional ubiquitins are conjugated successively onto the terminal ubiquitin already conjugated to the target protein to form polyubiquitin chains. The polyubiquitin chains have been velieved to be generated by iso-peptide bond formation between the C-terminal carboxyl group of one ubiquitin and an $\varepsilon$-amino group of one of seven Lys residues in another ubiquitin. In the case of the ubiquitin proteolytic pathway, polyubiquitin chains are conjugated via K48. K63 conjugated chains are involved in signaling or DNA repair without the conjugated protein being subject to degradation. However, the linear ubiquitin chain, as reviewed here, is unique because it is generated by peptide bond formation between the C-terminus of one ubiquitin and the $\alpha$-amino group of Met1 residues in another ubiquitin. Conjugated ubiquitins are cleaved by deubiquitinating enzymes (DUBs). The function of conjugated proteins is modulated via specific recognition of conjugated polyubiquitin chains 
complex that specifically generates linear polyubiquitin chains and named it as the linear ubiquitin chain assembly complex (LUBAC) [4]. Further analysis has revealed that linear polyubiquitination is involved in NF- $\mathrm{KB}$ activation [5].

\section{The Canonical NF-кB Activation Pathways}

$\mathrm{NF}-\kappa \mathrm{B}$ is a transcription factor that is involved in a broad array of biological phenomena including cell survival, inflammation, and innate and acquired immune responses [6]. Since aberrant activation of NF- $\kappa \mathrm{B}$ is reported in numerous pathological conditions including autoimmune diseases and neoplasms, the NF- $\mathrm{KB}$ activation pathway has been extensively studied. NF- $\mathrm{BB}$ activation pathways are basically subdivided into two distinct pathways, known as the canonical and alternative pathways [6]. The canonical NF- $\kappa \mathrm{B}$ activation pathway is discussed here since LUBAC-mediated linear polyubiquitination is known to be involved in the pathway [7]. NF- $\mathrm{\kappa B}$ is inactive in resting cells as it resides in the cytoplasm bound

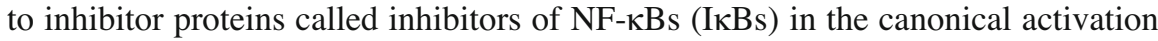
pathway (Fig. 2). The IкB kinase (IKK) complex, which comprises IKK1, IKK2, and NF- $\kappa \mathrm{B}$ essential modulator (NEMO), is activated upon stimulation with various agents such as Toll-like receptor (TLR) ligands or inflammatory cytokines including

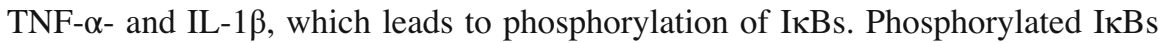
are recognized by the $\mathrm{SCF}^{\beta \mathrm{TrCP}}$ ubiquitin ligase and conjugated with $\mathrm{K} 48$ chains, which leads to phosphorylated IкBs to degradation by the proteasome. Then, liberalized NF- $\kappa \mathrm{B}$ translocates to the nucleus to induce the transcription of target genes (Fig. 2) [6]. Although the precise mechanism leading to the activation of the IKK complex has not been completely solved, K63 polyubiquitin chains have been shown to be involved in the IKK activation in the canonical pathway [8]. Since roles of K63 chains in NF- $\mathrm{KB}$ activation is not the main topic of in this article [8], the current hypothesis of the roles of K63 chains in the TNF- $\alpha$ - and IL- $1 \beta$-induced canonical NF- $\kappa \mathrm{B}$ activation pathway will only be summarized here. Upon binding TNF- $\alpha$ in addition to adaptor molecules, ubiquitin ligases including TRAF2 and cIAPs are recruited to the TNF receptor 1 (TNFR1) and K63 chains conjugated mainly onto RIP1. In the case of IL-1 $\beta$ signaling, TRAF6 E3 recruited to the IL-1 receptor (IL-1R) complex generates K63 chains on TRAF6 itself and on IRAK1. K63 chains, generated in the activated receptor complex, recruit the TAK1-TAB1TAB2/3 complex and the IKK complex via K63-selective binding of TAB2/3 or NEMO, respectively. TAK1 then phosphorylates specific Ser residues of IKK2, which leads to the phosphorylation and degradation of IкBs [8]. However, the involvement of K63-linked chains in NF- $\mathrm{B}$ activation has been challenged. In cells isolated from $\mathrm{KO}$ mice of Ubc13, a crucial component of an Ubc13-Uev1a E2 complex to generate K63 chains specifically, NF- $\kappa \mathrm{B}$ activation mediated by TNF- $\alpha$ is not overtly affected, although TNF- $\alpha$-mediated activation of MAPKs by these stimuli is severely impaired [9]. Moreover, K63 chains are dispensable for TNF- $\alpha-$, but not for IL-1 $\beta$-, induced canonical NF-KB activation [10]. Thus, although K63 


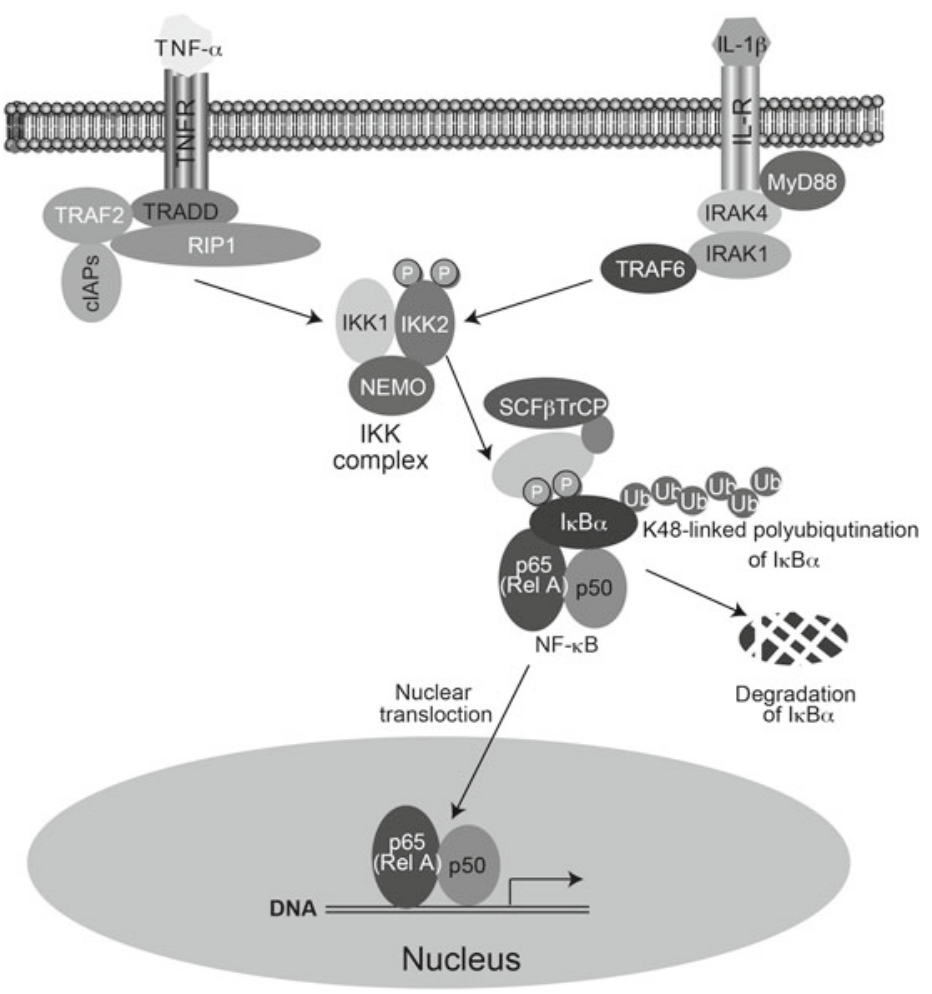

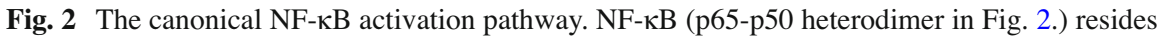
within the cytoplasm of resting cells in a form bound to the inhibitor protein, I $\mathrm{B} B \alpha$. Upon binding to TNF- $\alpha$, TRADD and RIP1 are recruited to the TNF receptor 1 (TNFR1). TRADD then recruits TRAF2 and cIAPs. In the case of IL- $1 \beta$ signaling, MyD88 is recruited to the IL-1 receptor (IL-1R), which recruits IRAK1 and IRAK4 to the receptor. IRAK1 binds to TRAF6. The ubiquitin ligase activities of TRAF6 and cIAPs are involved in the signaling cascade leading to canonical IKK activation, namely IKK2 phosphorylation. Phosphorylated I $\kappa \mathrm{B} \alpha$ is then conjugated with K48linked polyubiquitin chains by the $\mathrm{SCF}^{\beta T r C P}$ ubiquitin ligase and degraded by the proteasome. Liberated NF- $\mathrm{KB}$ translocates to the nucleus and induces the expression of target genes

chains play crucial roles in signaling, they might be dispensable for the canonical $\mathrm{NF}-\kappa \mathrm{B}$ activation, at least on some occasions including TNF- $\alpha$ stimulation.

\section{The Linear Ubiquitin Chains and Their Roles in NF-кB Activation}

One of the important features of the ubiquitin conjugation system is the diversity of the polyubiquitin chains [11]. Ubiquitin chains have been believed to be generated by conjugating $\mathrm{C}$-terminal carboxyl groups of ubiquitin to $\varepsilon$-amino groups of one of seven Lys residues of another ubiquitin. Although polyubiquitin chains are 
HOIL-1L

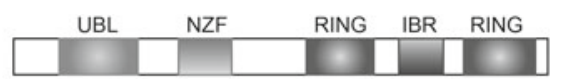

HOIP

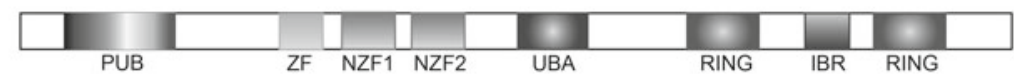

SHARPIN

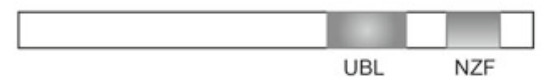

Fig. 3 Schematic representation of LUBAC, which is composed of HOIL-1L, HOIP, and SHARPIN. The zinc finger and RING-IBR-RING domains of HOIP are the substrate-binding site and $\mathrm{E} 3$ active site, respectively. IBR in-between RING, $N Z F$ Npl4-type zinc finger, $P U B$ peptide:Nglycanase/UBA- or UBX-containing proteins, $R I N G$ really interesting new gene, $U B A$ ubiquitinassociated domains, $U B L$ ubiquitin-like, $Z F$ zinc finger

generated by the repetitive functions of three enzymes, E1, E2, and E3, molecular mechanisms underlying polyubiquitin chain generation has not been completely solved [11]. During the dissection of the mechanism underscoring polyubiquitin generation, we have identified a new type of ubiquitin chain called the linear ubiquitin chain, in which the $\mathrm{C}$-terminal carboxyl group of ubiquitin is conjugated to the $\alpha$-amino group of the N-terminus of another ubiquitin [11]. Here, how the linear chains were discovered is discussed. We have identified the E3 complex composed of two RING-IBR-RING proteins, HOIL-1L and HOIP, and realized that HOIL-1L and HOIP possess multiple ubiquitin binding sites in addition to E2 binding sites (Fig. 3) [4, 12]. We thus hypothesized that the ubiquitin ligase complex recognizes ubiquitin as a substrate and conjugates ubiquitin onto it to generate polyubiquitin chains. Further analyses revealed that the HOIL-1L-HOIP complex indeed generates linear polyubiquitin chains exclusively [4].

During the course of seeking the physiological function, we found that siRNAmediated suppression of HOIP attenuated TNF- $\alpha$-mediated NF- $\kappa$ B activation [5]. In primary hepatocytes isolated from mice lacking HOIL- $1 \mathrm{~L}, \mathrm{TNF}-\alpha$-induced nuclear localization of $\mathrm{p} 65$, a subunit of $\mathrm{NF}-\kappa \mathrm{B}$, is heavily impaired. Moreover, TNF- $\alpha$-induced IKK activation and expression of target genes of NF- $\kappa \mathrm{B}$ are severely attenuated in embryonic fibroblasts (MEFs) from the HOIL-1L KO mice [5].

However, although deletion of molecules essential for NF- $\kappa \mathrm{B}$ activation, such as NEMO or IKK2, is embryonic lethal in mice, HOIL-1L KO is viable. HOIP, which is a catalytic subunit of the complex, was heavily decreased but not completely absent in HOIL-1L KO cells, which suggested that HOIP may have another binding partner besides HOIL-1L, and we identified SHARPIN as a binding partner of HOIP. The C-terminus half of SHARPIN exhibits significant homology with the N-terminal half of HOIL-1L, which is essential for binding to HOIP (Fig. 3) [13]. Further analyses revealed that SHARPIN forms the tertiary complex with HOIL-1L and HOIP [13]. The complex composed of HOIL-1L, HOIP, and SHARPIN conjugates to linear polyubiquitin chains, and we designated the complex composed of HOIL-1L, HOIP, and SHARPIN as LUBAC (Fig. 3) [13]. 
Pathophysiological roles of linear polyubiquitin chains were revealed by the analyses of spontaneous mutant mice called chronic proliferative dermatitis in mice (cpdm) [13-15]. Loss of SHARPIN is causative in cpdm mice that exhibit chronic inflammation including chronic dermatitis and immunodeficiency. In cells isolated from cpdm mice, the amount of the other components of LUBAC, HOIL-1L and HOIP, was reduced drastically by the lack of SHARPIN, thereby attenuating canonical NF- $\kappa \mathrm{B}$ activation induced by several stimuli including TNF- $\alpha$ and CD40 [13-15]. Recently, loss-of-function mutation of HOIL-1L provoked a fatal human inherited disorder characterized by chronic autoinflammation, invasive bacterial infections, and muscular amylopectinosis [16]. Loss of HOIL-1L or SHARPIN destabilizes the other two components of LUBAC and thereby suppresses signalinduced NF- $\mathrm{KB}$ activation [5, 13-15]. Thus, loss of regulatory subunits of LUBAC suppresses signal-induced canonical NF- $\kappa \mathrm{B}$ activation and provokes diseases characterized by autoinflammatory and immunodeficiency phenotypes, at least in part [7].

\section{Mechanism Underlying Linear Ubiquitin Chain-Mediated NF-кB Activation}

The ubiquitin conjugation system regulates a wide variety of physiological phenomena by conjugating polyubiquitin chains in a timely and selective manner [3]. The E3 enzymes must recognize target proteins prior to their ubiquitination [3]. In the case of LUBAC-mediated canonical NF- $\kappa \mathrm{B}$ activation, NEMO, an integral subunit of the IKK complex, is the substrate of LUBAC, and linear polyubiquitination of NEMO is shown to be involved in the activation of IKK that leads to canonical NF-кB activation [6]. Recent analyses revealed the molecular mechanism of linear polyubiquitination of NEMO-mediated IKK activation [17]. Upon stimulation with agents such as TNF- $\alpha$, LUBAC recognizes NEMO using the Npl4-type zinc finger 1 (NZF1) domain of HOIP (Fig. 4) and conjugates linear ubiquitin chains onto the proteins. Conjugation of di-ubiquitin (dimer) of linear linkage to NEMO appears enough to activate the IKK complex [17]. Activation of the IKK complex is mediated by the phosphorylation of IKK2 [6]. In general, phosphorylation of kinases is mediated either by trans-autophosphorylation or by upstream kinases [18]. The crystal structural analyses of human IKK2 revealed that homotypic interaction of the IKK2 kinase domain is crucial for IKK2 activation [19]. Moreover, NEMO possesses the ubiquitin binding activity that prefers linear di-ubiquitin, and NEMO's ubiquitin binding activity is critical for IKK activation [20]. We thus showed that linear di-ubiquitin conjugated to NEMO is recognized by another NEMO in trans, which triggers dimerization of the IKK complex and subsequent trans-autophosphorylation of IKK2 (Fig. 4) [17]. 
Fig. 4 The proposed role of linear chains in canonical $\mathrm{NF}-\kappa \mathrm{B}$ activation. NEMO, an integral component of the IKK complex, is recognized by the NZF1 of HOIP upon stimulation and is linearly ubiquitinated by

LUBAC. Linear di-ubiquitin is sufficient to activate IKK. Linear di-ubiquitin conjugated to NEMO is recognized by NEMO in another IKK complex in trans, triggering dimerization and trans-autophosphorylation of IKK2. Activated IKK2 phosphorylates $\mathrm{I} \kappa \mathrm{B} \alpha$, activating the canonical $\mathrm{NF}-\kappa \mathrm{B}$ pathway

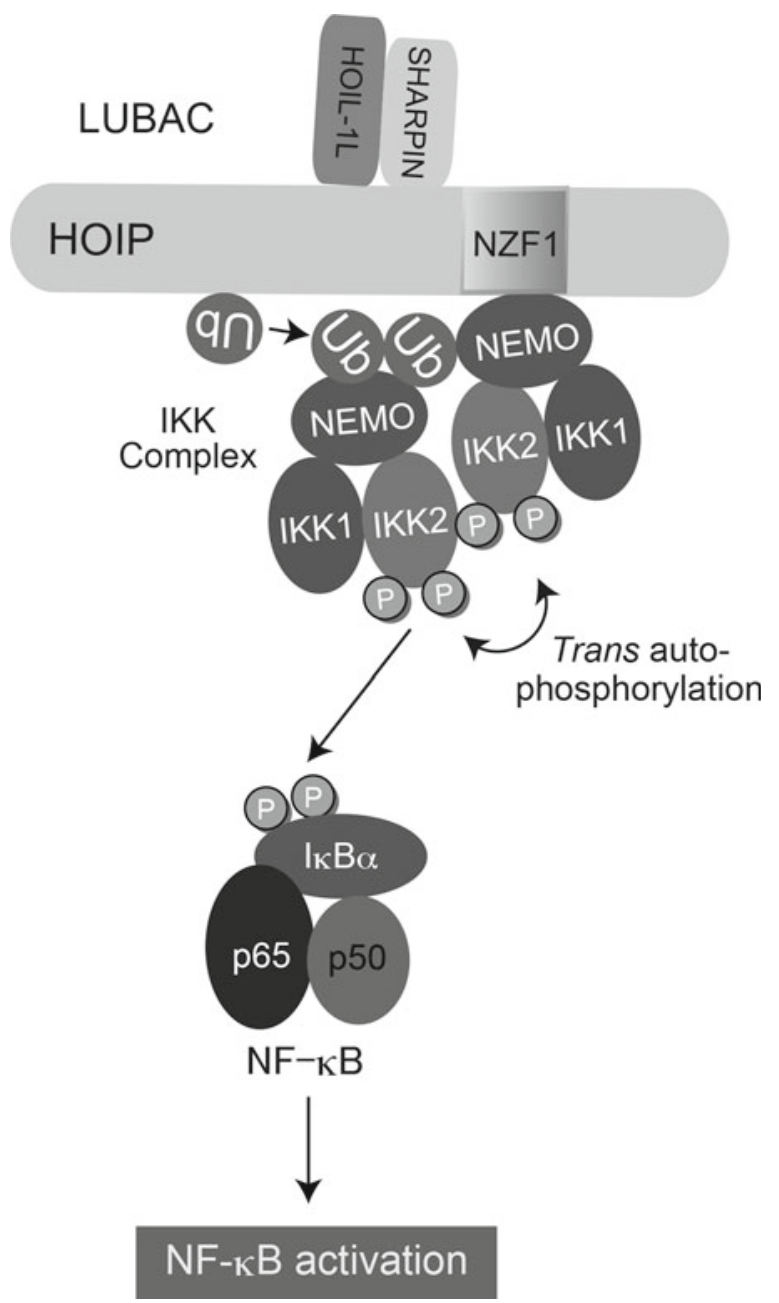

\section{Perspectives}

The involvement of LUBAC-mediated linear polyubiquitination in disease has been shown recently. Aberrant NF- $\kappa \mathrm{B}$ activation plays an essential role in the pathogenesis of activated B cell-like diffuse large B cell lymphomas (ABC DLBCL) [21]. Recent analyses showed that LUBAC is essential for the growth of ABC DLBCL cells through suppression of NF-KB activity. Moreover, two rare SNPs in the HOIP gene are enriched in ABC DLBCL [21]. We have also observed that attenuation of LUBAC suppressed lung metastases of osteosarcomas in mice [22]. Moreover, HOIP attenuates apoptosis induced by platinum-based genotoxins, including cisplatin and carboplatin, that are widely used as anti-cancer drugs [23]. Thus, suppression of LUBAC activity might be a suitable target to control malignant tumors. We have 
identified a new type of ubiquitin modification, linear ubiquitination, during our analyses to address the basic question of how the ubiquitin system generates polyubiquitin chains. We hope our unexpected finding will lead to the development of new therapeutic agents to control cancer.

Open Access This chapter is distributed under the terms of the Creative Commons Attribution Noncommercial License, which permits any noncommercial use, distribution, and reproduction in any medium, provided the original author(s) and source are credited.

\section{References}

1. Ciechanover A, Hod Y, Hershko A (1978) A heat-stable polypeptide component of an ATP-dependent proteolytic system from reticulocytes. Biochem Biophys Res Commun 81(4):1100-1105. doi:0006-291X(78)91249-4 [pii]

2. Hershko A, Ciechanover A (1998) The ubiquitin system. Annu Rev Biochem 67:425-479. doi:10.1146/annurev.biochem.67.1.425

3. Deshaies RJ, Joazeiro CA (2009) RING domain E3 ubiquitin ligases. Annu Rev Biochem 78:399-434. doi:10.1146/annurev.biochem.78.101807.093809

4. Kirisako T, Kamei K, Murata S, Kato M, Fukumoto H, Kanie M, Sano S, Tokunaga F, Tanaka K, Iwai K (2006) A ubiquitin ligase complex assembles linear polyubiquitin chains. EMBO J 25(20):4877-4887. 7601360 [pii] doi:10.1038/sj.emboj.7601360

5. Tokunaga F, Sakata S, Saeki Y, Satomi Y, Kirisako T, Kamei K, Nakagawa T, Kato M, Murata S, Yamaoka S, Yamamoto M, Akira S, Takao T, Tanaka K, Iwai K (2009) Involvement of linear polyubiquitylation of NEMO in NF-אB activation. Nat Cell Biol 11(2):123-132. doi:ncb1821 [pii] 10.1038/ncb1821

6. Hayden MS, Ghosh S (2008) Shared principles in NF-kB signaling. Cell 132(3):344-362. doi:S0092-8674(08)00120-7 [pii] 10.1016/j.cell.2008.01.020

7. Iwai K (2012) Diverse ubiquitin signaling in NF- $\kappa B$ activation. Trends Cell Biol 22(7):355-364. doi:10.1016/j.tcb.2012.04.001

8. Chiu YH, Zhao M, Chen ZJ (2009) Ubiquitin in NF-кB signaling. Chem Rev 109(4):1549-1560. doi:10.1021/cr800554j

9. Yamamoto M, Okamoto T, Takeda K, Sato S, Sanjo H, Uematsu S, Saitoh T, Yamamoto N, Sakurai H, Ishii KJ, Yamaoka S, Kawai T, Matsuura Y, Takeuchi O, Akira S (2006) Key function for the Ubc13 E2 ubiquitin-conjugating enzyme in immune receptor signaling. Nat Immunol 7(9):962-970. doi:. doi:ni1367 [pii] 10.1038/ni1367

10. Xu M, Skaug B, Zeng W, Chen ZJ (2009) A ubiquitin replacement strategy in human cells reveals distinct mechanisms of IKK activation by TNF $\alpha$ and IL-1 $\beta$. Mol Cell 36(2):302-314

11. Hochstrasser M (2006) Lingering mysteries of ubiquitin-chain assembly. Cell 124(1):27-34. doi:S0092-8674(05)01466-2 [pii] 10.1016/j.cell.2005.12.025

12. Yamanaka K, Ishikawa H, Megumi Y, Tokunaga F, Kanie M, Rouault TA, Morishima I, Minato $\mathrm{N}$, Ishimori K, Iwai K (2003) Identification of the ubiquitin-protein ligase that recognizes oxidized IRP2. Nat Cell Biol 5(4):336-340. doi:10.1038/ncb952 ncb952 [pii] 10.1038/ncb952

13. Tokunaga F, Nakagawa T, Nakahara M, Saeki Y, Taniguchi M, Sakata S, Tanaka K, Nakano H, Iwai K (2011) SHARPIN is a component of the NF-kB-activating linear ubiquitin chain assembly complex. Nature 471(7340):633-636. doi:nature09815 [pii] 10.1038/nature09815

14. Gerlach B, Cordier SM, Schmukle AC, Emmerich CH, Rieser E, Haas TL, Webb AI, Rickard JA, Anderton H, Wong WW, Nachbur U, Gangoda L, Warnken U, Purcell AW, Silke J, Walczak H (2011) Linear ubiquitination prevents inflammation and regulates immune signalling. Nature 471(7340):591-596. doi:nature09816 [pii] 10.1038/nature09816 
15. Ikeda F, Deribe YL, Skanland SS, Stieglitz B, Grabbe C, Franz-Wachtel M, van Wijk SJ, Goswami P, Nagy V, Terzic J, Tokunaga F, Androulidaki A, Nakagawa T, Pasparakis M, Iwai K, Sundberg JP, Schaefer L, Rittinger K, Macek B, Dikic I (2011) SHARPIN forms a linear ubiquitin ligase complex regulating NF-кB activity and apoptosis. Nature 471(7340):637-641. doi:nature09814 [pii] 10.1038/nature09814

16. Boisson B, Laplantine E, Prando C, Giliani S, Israelsson E, Xu Z, Abhyankar A, Israel L, Trevejo-Nunez G, Bogunovic D, Cepika AM, Macduff D, Chrabieh M, Hubeau M, Bajolle F, Debre M, Mazzolari E, Vairo D, Agou F, Virgin HW, Bossuyt X, Rambaud C, Facchetti F, Bonnet D, Quartier P, Fournet JC, Pascual V, Chaussabel D, Notarangelo LD, Puel A, Israel A, Casanova JL, Picard C (2012) Immunodeficiency, autoinflammation and amylopectinosis in humans with inherited HOIL-1 and LUBAC deficiency. Nat Immunol 13(12):1178-1186. doi:10.1038/ni.2457

17. Fujita H, Rahighi S, Akita M, Kato R, Sasaki Y, Wakatsuki S, Iwai K (2014) Mechanism underlying IкB kinase activation mediated by the linear ubiquitin chain assembly complex. Mol Cell Biol 34(7):1322-1335. doi:10.1128/MCB.01538-13

18. Hunter T, Lindberg RA, Middlemas DS, Tracy S, van der Geer P (1992) Receptor protein tyrosine kinases and phosphatases. Cold Spring Harb Symp Quant Biol 57:25-41

19. Polley S, Huang DB, Hauenstein AV, Fusco AJ, Zhong X, Vu D, Schrofelbauer B, Kim Y, Hoffmann A, Verma IM, Ghosh G, Huxford T (2013) A structural basis for IkB kinase 2 activation via oligomerization-dependent trans auto-phosphorylation. PLoS Biol 11(6):e1001581. doi:10.1371/journal.pbio.1001581

20. Rahighi S, Ikeda F, Kawasaki M, Akutsu M, Suzuki N, Kato R, Kensche T, Uejima T, Bloor S, Komander D, Randow F, Wakatsuki S, Dikic I (2009) Specific recognition of linear ubiquitin chains by NEMO is important for NF-KB activation. Cell 136(6):1098-1109. doi:S00928674(09)00264-5 [pii] 10.1016/j.cell.2009.03.007

21. Yang Y, Schmitz R, Mitala J, Whiting A, Xiao W, Ceribelli M, Wright GW, Zhao H, Xu W, Rosenwald A, Ott G, Gascoyne RD, Connors JM, Rimsza LM, Campo E, Jaffe ES, Delabie J, Smeland EB, Braziel RM, Tubbs RR, Cook JR, Weisenburger DD, Chan WC, Wiestner A, Kruhlak MJ, Iwai K, Bernal F, Staudt LM (2014) Essential role of the linear ubiquitin chain assembly complex in lymphoma revealed by rare germline polymorphisms. Cancer Discov 4(4):480-493. doi:10.1158/2159-8290.CD-13-0915

22. Tomonaga M, Hashimoto N, Tokunaga F, Onishi M, Myoui A, Yoshikawa H, Iwai K (2012) Activation of nuclear factor-kappa B by linear ubiquitin chain assembly complex contributes to lung metastasis of osteosarcoma cells. Int J Oncol 40(2):409-417. doi:10.3892/ijo.2011.1209

23. Mackay C, Carroll E, Ibrahim AF, Garg A, Inman GJ, Hay RT, Alpi AF (2014) E3 ubiquitin ligase HOIP attenuates apoptotic cell death induced by cisplatin. Cancer Res 74(8):2246-2257. doi:10.1158/0008-5472.CAN-13-2131 